\title{
PROGRAMA DE SOCIALIZACIÓN ECONÓMICA EN INTERDEPENDENCIAS EMPRESARIALES PARA EL EMPRENDIMIENTO DE TURISMO RURAL-VIVENCIAL EN CARAZ, ANCASH
}

\author{
ECONOMIC SOCIALIZATION PROGRAM FOR THE ENTERPRISE BUSINESS \\ INTERDEPENDENCIES OF RURAL TOURISM-EXPERIENTIAL IN CARAZ, ANCASH
}

\author{
Mildred Paredes T¹.; Héctor Hernandez V.; Luis Vicuña P.; Concepción Gonzalez G. \\ Universidad Nacional Mayor de San Marcos, Lima, Perù \\ (RECIBIDO 15/2/2012; ACEPTADO 25/6/2012)
}

\begin{abstract}
RESUMEN
Estudio con diseño cuasi experimental, evaluación antes y después, muestreo intencional de colaboradores voluntarios de barrios urbanos Asociados en APEMYPE Huaylas Caraz y de la zona rural la asociación SHOCSHA Vivencial convocados para responder: Cómo afecta el Programa de socialización económica en interdependencias empresariales al emprendimiento del turismo rural- vivencial en Caraz, Ancash. La data recogida mediante la Escala de Actitudes- (ESCAIETA-2011) expresa 0.86 de confiabilidad y la aplicacion de la Encuesta No.1 Conocimientos Turismo Alternativo (E1CTA-2011): Rural-Vivencial para el Programa de Capacitación. Se procesó y analizó los datos por estadígrafos no parametricos, de la variable dependiente (VD) Actitudes hacia las interdependencias empresariales para el emprendimiento de Turismo alternativo- Rural/ Vivencial, por efecto Variable independiente (VI) Talleres: Términos económicos de estrategia por alianzas en gestión empresarial e institucional y la variable de control (VC) Condición de actividades de turismo vivencial centradas en el rescate de productos culturales (UNESCO 2005). Previo diagnóstico de probables emprendedores por juntas vecinales e información del proyecto, se formaron dos grupos de intervención. observándose confusión sobre las modalidades del turismo previamente a la aplicación de ESCAIETA-2011 (antes y después de la aplicación de la VI, 9 talleres). Los resultados de la investigación encuentra que las actitudes hacia la interdependencia empresarial para el emprendimiento son positivas, con una tendencia al incremento, sin llegar a marcar una diferencia significativa ni pasar a muy positiva, siendo expreso que sus actividades en el sector turismo no les redita utilidad, por contrario, es de mayor porcentaje el de aventura. Se acepta la hipótesis nula, el Programa de Socialización Económica en Interdependencias Empresariales e Institucionales para orientar el emprendimiento del turismo rural-vivencial en Caraz, Ancash no afecta.
\end{abstract}

Palabras clave: Actitudes turismo alternativo, interdependencia para el emprendimiento,

1 Docente Asociada de la facultad de psicología UNMSM. E-mail: mildredparedestarazona@gmail.com 
APEMYPE, Shocsha Vivencial.

\begin{abstract}
Quasi-experimental study design, evaluation before and after intentional sample of volunteers from urban neighborhoods Associates Huaylas APEMYPE Caraz and rural Asociacion SHOCSHAVivencial to answer: How it affects the economic socialization program interdependencies to entrepreneurship in tourism business rural-experiential in Caraz, Ancash.

DATA collected by Attitude Scale-(ESCAIETA-2011) of 0.86 for reliability and Knowledge Survey No.1 Alternative Tourism (E1CTA-2011): Rural-to Experiential Training Program. Was processed and analyzed the data by statisticians not parametric, dependent variable (DV) Attitudes towards enterprise interdependencies for alternative tourism venture, Rural / Experiential, Independent Variable effect of (VI) Workshops: Economic Terms partnerships Strategy business and institutional management and the control variable (CV) Status of experiential tourism activities focused on the rescue of cultural products (UNESCO 2005).

Previous diagnosis of probable entrepreneurs by neighborhood groups and information of the project, agreed to two intervention groups. Observed confusion on the modalities of Tourism before the application of ESCAIETA-2011 (before and after application of the VI, 9 workshops).
\end{abstract}

Attitudes towards business Interdependence for entrepreneurship are positive, with an increasing trend, short make a significant difference or a very positive move, being expressed that their activities in the tourism sector not for profit REDITA opposite is more share the adventure.

Null hypothesis is accepted, the Program of economic socialization in business and institutional interdependencies to guide the rural-tourism venture in experiential Caraz, Ancash not affected.

Keywords: Attitudes alternative tourism, interdependence for entrepreneurship, APEMYPE, Asociación Shocsha Vivencial.

\title{
INTRODUCCIÓN
}

El conocimento académico, producto de la investigación de los últimos seis años en el tema, el que permite el avance de la psicología organizacional y económica para gestión de proyectos sociales, con el diseño de estos aportes en modelos y métodos para buscar una mejora en la calidad de vida en la zona, y evaluar posteriormente su posible replica con los ajustes necesarios en otros escenarios similares; en la conversión en unidades productivas en servicios de turismo rural y vivencial que les posibilite su articulación empresarial sostenible, tal como se observa en proyectos exitosos en otras comunidades rurales del país: Cusco, Puno, y experiencias recientes en Ayacucho y Huancavelica, Mincetur, ONG; y disminuir la tasa del error que lleva al fracaso, desesperanza y el mantenimiento de conductas dependientes luego que son dejadas o se retiran los organismos promotores de intervención social, experiencias que no han resuelto, sino han alimentando la dependencia que va unido a la pobreza.

El conocimiento difundido en revistas científicas y el trabajo de intervención ha posibilitado involucrar a la Gerencia de Educación y de Proyectos Productivos Rurales de la Municipalidad de Huaylas, Caraz, a participar en la zona, mediante 
la estrategia que se investiga para su transferencia, aunado a su planeamiento concertado; así mismo involucrar a las ONG que ejecuten tomando los aportes en sus intervenciones.

De esta manera se contribuye con los conocimientos significativos de la zona urbana y rural, en relación al emprendimiento y alfabetización económica en interdependencias empresariales (alianzas estratégicas) para el emprendimiento del turismo (turismo vivencial). A pesar que cognitivamente manifiestan su interés por el turismo, aún cuando sus actividades en el sector turismo no les reditúa utilidad de manera real, sus actitudes positivas hacia la interdependencia empresarial al emprendimiento hace posible formar articulaciones económicas.

En tanto el conocimiento que tienen contrasta con los beneficios que pueden obtener sostenidas por las actitudes positivas al sector del turismo posibilita asumir con responsabilidad continuar con el aporte a la comunidad científica de un programa de intervención en la zona urbana y rural de provincias, incorporando de manera activa la información prevista en el Plan Estratégico de Desarrollo Concertado de la Provincia de Huaylas 2007-2015. De manera específica contribuir con los objetivos de VRI en realizar la transferencia del conocimiento al país.

Complentariamente ha posibilitado mediante otras condiciones evaluar y desarrollar la implementación un modelo, como articular conceptos de alfabetización económica dentro de las resistencias locales, y conducirlos hacia el desarrollo de unidades productivas sostenibles, vinculando y armonizando lo tradicional con lo moderno; el diálogo y no la imposición en concordancia a la política de investigación de de la UNMSM: VRI, CSI, IIPSI y de organismos e instituciones como la UNESCO, BM, OIT, OMS, FAO, entre otros.

\section{Antecedentes}

La formación de conceptos básicos de economía aplicados a la gestión de unidades productivas en servicios turísticos, en el marco de la alfabetización económica en gestión, cuya estrategia se diseñe en la estructura vertical de servicios turísticos con la construcción o formación de articulaciones organizacionales que exprese comportamiento propio de las interdependencias empresariales. Así, se sustenta en los estudios previos referidos al emprendimiento en pobladores de la zona de intervención, tal como se concluye que hay un cambio en el grupo cuasi experimental en la segunda evaluación con respecto a la primera evaluación de la conducta de emprendimiento (al $0.05 \mathrm{de} \mathrm{t}=7.637$ ); conducta que se presenta influenciada por la intervención de los talleres de capacitación en alfabetización económica en términos básicos de economía aplicados a la gestión, proceso desarrollado en el marco de la socialización económica intergeneracional en los colaboradores quechuahablantes del caserío de Queral de Caraz, Ancash, (Paredes, M. y Colb., 2010 A).

Dicha transferencia de conceptos se desarrolla mediante la experiencia que pueden lograr los colaboradores al ejecutar actividades reñidas con la producción y la venta de chalinas, tareas que asumen el rol estímulos tangibles para relacionar y lograr 
la asociación con el concepto de economía. La chalina es un producto descrito y recuperado como uno de los cuales se le puede considerar una expresión cultural descrita entre los bienes a ser productos comerciables, según los lineamientos y recomendaciones que cumplen dichos bienes o servicios culturales planteados por la UNESCO en el 2005, Paredes, M. Y Colb. (2009), resaltando que la chalina es identificada como producto cultural por las colaboradoras del estudio y que están en proceso de otorgarle el valor agregado para su comercialización, en tanto el producto guarda signos gráficos y colores de su acervo cultural. Este bien cultural se le identifica como una alternativa para mejorar cualitativamente su economía de subsistencia, destacándose que los participantes colaboradores del estudio denotan en su comportamiento el conocimiento de que la chalina es un producto comercial y que puede coadyuvar a su economía doméstica, no solo incrementando su producción sino también proporcionando un contexto situacional con un ambiente favorable y de reto al constituir la Asociación de Productores Tucu Wain del Caserío de Queral Perú; tal como se señalaba que dicho emprendimiento en principio se dará mediante la producción de una línea de textil con el producto: chalinas, la que es consignada por sus respectivos agentes como alternativa factible y enriquecedora del recurso humano y capital social de la comunidad, a propósito de reducir la brecha de desigualdad en la distribución de recursos, PNUD (2010).

Acciones de desarrollo de recursos humanos y de implementación de la gestión de la organizaciones rurales sustentada en la construcción de la representación social organizacional, sui generis, y planteada en la perspectiva de que la transmisión intergeneracional de saberes, modelos que aproximan a proporcionar un marco también para la instrumentación de los principios del aprendizaje en la formación de conceptos básicos de economía aplicados a la gestión de unidades productivas, a propósito de la búsqueda de la efectividad del programa de alfabetización económica en el desarrollo de conductas de emprendimiento en pobladores quechua hablantes de Caraz, Ancash en Paredes, M. y Colb. (2010 A). Modelo que se está constituyendo un referendo para la Educación Económica, entendida como la "Acción educativa intencionada cuyo objetivo es facilitar que niños y adultos construyan nociones económicas básicas y desarrollen estrategias para tomar decisiones adecuadas que les permitan situarse ante la sociedad de consumo" Denegri, M. Colab (1999)

El Plan Estratégico de Desarrollo Concertado de la Provincia de Huaylas 20072015, plantea entre sus nuevos retos y responsabilidades la de conformar y fortalecer instancias especializadas en la gestión e implementación de planes de desarrollo, así como en los objetivos estratégicos para el eje turístico. Considera Fomentar y proponer la participación activa del gobierno local y de la sociedad civil en el desarrollo de dicha actividad, teniendo entre sus debilidades "Escaso interés de concertación y cooperación interinstitucional" así como que el "Desarrollo de las actividades humanas dañan el medio". Gobierno Local que avizora entre su competencia la responsabilidad social que al ejercerla se deba cimentar en base a la participación e involucramiento de sus ciudadanos como actores, en principio con 
calidad de vida física, socioeconómica, cultural, mental y psicológica bajo la óptica de los valores y la ética, partiendo del conocimiento de los intereses y naturaleza de sus estilos de vida, opiniones inherentes de la cultura de sus actores como su toma de decisiones para su inclusión, de ahí que se tiene que intervenir a nivel cognitivo iniciando en base del conocimiento, la formación de categorías que es la partida para los aprendizajes de actitudes, en este caso proactivas a la actividad turística, para la sostenibilidad de la conducta en el comportamiento económico.

Todo espacio sociocultural es sumamente complejo y delicado, pues las sensibilidades, las percepciones y los imaginarios difieren, lo que exige de los investigadores entender su lógica para interactuar de manera distendida y en confianza, requisitos necesarios para la transferencia de conceptos económicos, considerando el contexto de la comunidad rural andina, en tanto se ha sugerido que se observa conductas de evasión o rechazo a tareas de actividades educativas orientadas a la producción, siendo más bien que "en realidad las personas no se resisten al cambio, se resisten a que se les imponga el cambio" (Bregman, 2009). De ahí que se hace necesario identificar aquellos indicadores válidos para la interdependencia empresarial o articulaciones de unidades productivas por el mercado, como son las variables psicosociales: actitudes, percepciones, motivaciones; las que tienen importancia y posibilita el sostenimiento para que prevalezcan las negociaciones constituidas por interdependencias basadas en una negociación integrativa o cooperativa, "Yo gano, Tú ganas"; de ahí que las variables psicosociales juegan un rol tan igual que las variables: tecnologías, económicas o quizás mayores que las financieras por ser propiciadoras de alianzas estratégicas empresariales de mayor volatilidad que las de proceso productivo, razón que fortalece enfocar a las interdependencias empresariales como estrategia para la competitividad con calidad en la gestión (Paredes, 2001:111-132).

La articulación empresarial es una estrategia demostrada como válida para enfrentar lo complejo y competitivo que son la competencia en el mercado y los gustos del consumidor, en tanto proporciona, al constituirse, a sus asociados, elementos como los factores de la gestión, el conocimiento y la capacidad de oferta de servicios o bienes. Fortalecidos por variables como la percepción, actitudes y motivación; las que actúan como amalgamas en la interdependencia empresarial e institucional, entendida esta como una "estrategia de la gestión de calidad", Paredes, M. (2001). Asimismo, mediante dicha estrategia los actores con limitados recursos económicos y financieros pero con habilidades desarrolladas en la elaboración de sus expresiones culturales en productos, se ven posibilitados para responder eficazmente a las exigencias de superación de la situación de atraso y postergación, en tanto que son identificados como población vulnerable o excluida. Y que podrán conseguirlo interviniendo desde una perspectiva productiva, constituyendo unidades productivas de la articulación y estableciendo la cadena respectiva, la que es presentada como una modalidad de enfrentar los retos externos o del mercado, aprendiendo e implantar en su manera de gestionar sus escasos recursos económicos con el propósito de tener calidad de vida. 
Entre los antecedentes se cuenta con el aporte de Ho, P. (2011) quien examinó por primera vez la reacción de los residentes de Fenqifu (pueblo de montaña con senderos sinuosos en Taiwan) al impacto del turismo y evaluó la aceptabilidad de las estrategias de gestión. A través de este estudio se adentró aún más en el diagnóstico de los factores que podrían influir en las percepciones de las estrategias de gestión, el que suministro insights útiles para el establecimiento de estrategias de turismo sostenible. Tomó en cuenta que con el fin de alcanzar la sostenibilidad del destino turístico, era esencial la evaluación de diferentes estrategias de gestión de impactos, como se sustenta en Gu \& Wong (2006) tal que las percepciones de los residentes hacia el impacto de las estrategias de gestión de turismo sirvieron de referencia para la formulación de políticas.

Dichos residentes percibieron las estrategias de gestión ambiental más positivamente que los enfoques sociocultural y económico. La estrategia de gestión indirecta (mayor libertad al turista y trabajar con un poco control), fue mejor recibida que las estrategias de gestión directa (restricciones de la actividad, reforzamiento por la ley y presencia de la autoridad). Los residentes prefirieron las estrategias indirectas de gestión ambiental y no fueron apreciadas las estrategias económicas directas. Las estrategias de gestión de "establecer signos, mapas y el sistema de interpretación para guiar a los turistas" y "establecer puestos de primeros auxilios para la seguridad del turista" fueron calificadas muy positivamente por los residentes. La prestación de los signos eficaces y mapas de dirección evitan que los turistas se pierdan. Por otro lado, los residentes no apreciaron "cobrar por los ingresos extra." Este resultado sugiere que los residentes tenían miedo de disminuir la cantidad de turistas, imponiendo estrategias económicas directas (por ejemplo, el cobro de honorarios para aumentar los ingresos y el control de la cantidad de turistas). También implica que los residentes todavía valoraban el mito de la promoción de "turismo de masas". Este malentendido se opone al principio de la sostenibilidad del desarrollo turístico. Por lo tanto, la relación entre la gestión de la estrategia económica y los cambios de número de turistas pueden ser examinadas con el fin de aclarar inquietudes de los residentes.

También, Ho, P. investigó los factores que influyen en la percepción de los residentes de las estrategias de gestión del turismo de impacto. Sus hallazgos sugieren que las variables demográficas en la ocupación tuvieron impacto en la percepción de las estrategias de gestión. Los residentes que trabajan en los sectores de servicios/ negocio reconocieron altamente las estrategias de gestión de los residentes empleados en el sector de la agricultura y la silvicultura. La posible explicación para este hallazgo es que la gente que trabaja en los sectores de servicios / negocio pueda tener algún grado de relación con las actividades turísticas. En cuanto a los atributos de la distancia residencial con las actividades turísticas, los residentes que viven lejos de "Old Street" tuvieron menor reconocimiento de las estrategias de gestión que los residentes que viven cerca de dicha calle. Ya que "el apoyo al turismo" y "el conocimiento del turismo" están altamente correlacionados con la percepción de estrategias de gestión, los residentes deberían tener actitudes positi- 
vas hacia el desarrollo del turismo. Este hallazgo refleja también en su referencia de Andereck y Vogt del 2000, la proposición de que la actitud de los residentes hacia el turismo es un factor importante en las estrategias de desarrollo regional.

Desde una perspectiva de gestión, los resultados indicaron que los factores relacionados con el desarrollo del turismo y la participación en el turismo influyeron diferencialmente en la aceptación de las estrategias de gestión para el turismo y el impacto de la recreación. Los resultados también sugieren que los residentes Fenqifu es probable que reaccionen de manera diferente a las estrategias de gestión, de acuerdo con su ocupación y su actitud hacia el desarrollo del turismo. Proporcionar más información sobre el desarrollo del turismo a los residentes sería la estrategia que rinda más apoyo para la gestión de los impactos del turismo. Los residentes que se han comprometido con el desarrollo del turismo o a tener una actitud positiva hacia las actividades turísticas reciben bien las estrategias de gestión. Este hallazgo tiene el potencial de informar y reflejar con mayor precisión la importancia del apoyo local para el desarrollo del turismo y hacer frente a los impactos de recreación. Los gerentes de áreas de Fenqifu están alentados a la difusión del turismo y la educación ambiental para reforzar los reconocimientos de los residentes a las estrategias de gestión.

$\mathrm{Al}$ respecto del desarrollo sostenible Ho, P. lo señala como un objetivo ideal para destino de turismo, especialmente por el valor de naturaleza y áreas de cultura. El principio fundamental del desarrollo sostenible es buscar una mejor solución que llegue al desarrollo de calidad, ahora y para la futura generación. De ahí, es esencial dirigir los impactos potenciales del desarrollo de turismo, acercando sistemáticamente el valor de la actitud a estrategias de dirección de impacto de turismo de los residentes. También los resultados de la investigación muestran que los vecinos están más preocupados por los cambios negativos en las condiciones ambientales, aún cuando dieron la bienvenida a los resultados seguros de la mejora económica. Las conclusiones revelaron que los vecinos preferían las estrategias de dirección desviadas para evitar ofender a visitas por reglas directas. Definitivamente, algunos factores críticos, que pueden influir en las impresión de impactos de turismo y las aprobaciones de las estrategias de dirección de impacto, fueron revisados para proveer la perspicacia las explicaciones para el turismo afectan la dirección.

Allen, L. R., Hafer, H. R., Long, P. T., \& Perdue, R. R. (1993) Encontraron que las actitudes hacia la recreación y el desarrollo del turismo en residentes rurales de 10 ciudades de Colorado (USA), se presentaron de la siguiente manera: las comunidades en que su nivel económico correspondía con el nivel de desarrollo de turismo (bajo/bajo y alto/alto) mostraron mejores actitudes hacia el desarrollo del turismo que aquellas comunidades en las que se daban relaciones opuestas (bajo/ alto y alto/bajo). Más aún, las actitudes hacia la recreación fueron afectadas por el nivel económico pero no por el nivel de desarrollo del turismo y adicionalmente, el tiempo de residencia no afectó a las actitudes hacia el desarrollo del turismo.

Así mismo se da como ejemplo que hay que adicionar otros servicios que resulte 
atractivo para los miembros de los diferentes grupos de familia de un circuito turismo alternativo, Howley, M., \& Westering, J. v. (2008) demostró que el desarrollo del turismo de vino, tiene un seguro efecto posible para áreas (de Texas y Rioja- Inglaterra) de cultivadores y productores de vino. Refiriéndose a que los productores vinícolas, en su mayor parte, no son organizados para obtener el beneficio máximo del turismo de vino. Actualmente las viñas inglesas están padeciendo de la depresión general en agricultura británica y los propietarios de la viña se beneficiarían de cualquier ganancia adicional como podía ser las generadas por el gasto del turista. Un estudio cualitativo en 2001 y 2002 indica que las actitudes al turismo de vino de propietarios de viñas y las actitudes que identifican maneras en que piensan que es el turismo de vino en el Reino Unido, posibilita a ser apoyados y organizados en beneficio de los productores de vino. Así la cada vez más la actitud profesional de productores de vino inglés en el 2000 fue reconocida por instituciones públicas, como el Ministerio de Agricultura, Pesca y Alimentación que reconoció por primera vez, este tipo de industria, hecho que llevo a las dos asociaciones profesionales a unirse y crear varios planes de levantar tanto la calidad como el perfil de vino inglés.

Se menciona también en el estudio de Howley, M., \& Westering, J. v, una alternativa en una ruta o paquete planificado que incluye la ruta del vino, se tiene los típicos ejemplos de otras atracciones como son sitios de herencia, granjas de queso, hoteles, restaurantes y bares. Por lo tanto, una fiesta de turistas podría ser llevada a cabo en una viña inglesa, entonces luego visitan una granja de queso, sigue luego para un almuerzo pre-planeado de productos locales en un restaurante, visitando una sitio de herencia después, y de quedarse definitivamente over in un hotel rural que era también parte del plan. Los Tour de esta naturaleza son organizados por el centro de vino inglés, en una operación de venta al por mayor y mercadotecnia comercial.

MP Jale Aliyeva (2011) como miembro del comité de cultura parlamentario azerbaiyano propone que debe darse una actitud especial a la promoción de la historia del país en el proyecto de ley "En el turismo" próximo de Azerbaiyán, como "la familiarización de extranjeros con la historia azerbaiyana debe ser uno aspecto muy importante parte del turismo".

Gursoy, D., Chi, C. G., \& Dyer, P. (2010), revisan las actitudes hacia dos tipos diferentes de desarrollo del turismo, el turismo masivo y el turismo alternativo, usando datos tomados de residentes vecinos de Coast Sunshine, Australia. Las conclusiones de estudio revelan que el soporte que la comunidad anfitriona está afectado directamente y/o indirectamente por el nivel de sentimiento de comunidad, la anexión a la comunidad, los valores ecocéntricos, el uso de la base de recursos de turismo, el estado de la economía local, y el impacto percibido del desarrollo de turismo. Las conclusiones indican aunque algunos de los factores influían en las actitudes hacia el turismo tanto masivo como alternativo, es aparente las actitudes hacia la forma del desarrollo para ser formada la base de la percepción de diferentes factores del turismo. 


\section{Hipótesis}

Afecta el programa de socialización económica en interdependencias empresariales e institucionales para orientar el emprendimiento del turismo rural-vivencial en Caraz, Ancash.

\section{Objetivos}

Identificar la tendencia de la dirección de actitudes antes y después hacia la interdependencia para el empoderamiento luego de la aplicación del programa de socialización económica en el grupo cuasi experimental

Comprobar el emprendimiento luego del programa de socialización mediante la interdependencia empresarial e institucional.

\section{Metas Específicas}

Se ha intervenido en dos asociaciones, no se ha logrado formar una unidad productiva asociativa en servicios de turismo vivencial a su interior.

Se ha icrementado en un $25 \%$ los conceptos de interdependencia económica aplicada a la gestión de calidad.

Se ha construido la escala de actitudes hacia la interdependencia empresarial e institucional para el emprendimiento.

Diseño de nueve talleres sobre emprendimiento.

\section{MÉTODO}

Es una investigación del tipo experimental, con un diseño cuasi experimental antes y después con dos grupos voluntarios; para orientar el aprendizaje a la tendencia positiva de las actitudes hacia las interdependencias empresariales e institucionales y buscar el implante de las conductas de emprendimiento en los colaboradores de Caraz, Ancash.

\section{Diseño}

Diseño cuasi experimental, medición antes y después entre un periodo de cinco meses en dos grupos de colaboradores voluntarios, utilizará el cuestionario de conocimiento de saberes y una Escala de actitudes para la medición previa y luego de aplicar los talleres de capacitación a los dos grupos experimentales; una de la zona urbana y otra de zona rural de la ciudad de Caraz. Así mismo, se empleará como medio de experiencia o condición concreta la técnica que desarrollan durante las actividades propias que describen el proceso de elaboración, producción o confección de sus bienes o servicios preferentemente aquellos productos culturales 
en paquetes turísticos o complementarios de una ruta turística de acuerdo con la UNESCO (2005) en proyectos productivos de servicios turísticos. Los mismos son los contenidos del Programa de Capacitación en conceptos de economía para la gestión de calidad que tiene como estrategia las interdependencias empresariales.

\section{Población}

Los pobladores mayores de 18 años de las juntas vecinales correspondientes a barrios de Caraz que constituyen la Asociación de APEMYPE Huaylas Caraz y de la zona rural la Asociación Shocsha Vivencial, conforman el universo poblacional. La muestra será determinada intencionalmente por decisión voluntaria de cada asociación.

\section{Muestra}

Tabla 1: Muestra inicial en función de la procedencia, urbana y rural

\begin{tabular}{ccc}
\hline Urbano & Rural & Total \\
\hline 25 & 4 & 29 \\
\hline
\end{tabular}

Tabla 2: Distribución de las muestras experimental según el sexo

\begin{tabular}{cccc}
\hline & Varones & Mujeres & Total \\
\hline APEMYPE & 7 & 3 & 10 \\
\hline Shoksha & 2 & 2 & 4 \\
\hline
\end{tabular}

\section{Proceso}

1. Formalización de ejecución del proyecto de investigación con los dirigentes de los barrios de Caraz y comunidad campesina de acceso a Caraz: APEMYPE Huaylas Caraz y de la zona rural la Asociación Shocsha Vivencial. Actividad compleja de ida y venida por la falta de toma de decisiones de los colaboradores. Se logró formalizar luego de 5 meses.

2. Formulación del marco teórico.

3. Descripción de actividades productivas con los productos culturales de la zona: Barrio Urbano y Rural del Estudio.

4. Elaboración perfil psicográfico y etnográfico de los grupos de asociados.

5. Construcción de instrumentos de recolección de datos: Registro de Observación de conductas de empoderamiento.

6. Identificación y acuerdo de su participación, realizada a los cinco meses, luego de explorar seis grupos u unidades convocadas por la Municipalidad de Huaylas Caraz. 
7. Medición Antes: De la variable dependiente (VD) luego de formalizar con los colaboradores su participación.

8. Intervención con aplicación de la VI: consistente en 9 talleres del Programa de Socialización económica con adultos, en la perspectiva de la transmisión intergeneracional durante 5 meses.

9. Medición Después: VD, se realizó luego de aplicar los 9 tallares del Programa de Capacitación, a los seis meses de la primera evaluación.

10. Análisis y discusión de la DATA.

11. Evaluación e Informe.

12. Retroalimentación con los colaboradores participantes.

\section{instrumentos}

1. Escala de Actitudes hacia las interdependencias empresariales para el emprendimiento de Turismo alternativo- Rural/ Vivencial- (ESCAIETA-2011) con la confiabiliodad de 0,84 y validez de contenido por poder de discriminación entre grupo superior y grupo inferior, al 0,05 .

2. Encuesta N. ${ }^{\circ} 1$ de Turismo Alternativo: Rural-Vivencial para el Programa de Capacitación, E1CTA-2011. Ver Anexo N. ${ }^{\circ} 2$

3. La observación de conductas de emprendimiento durante el desarrollo de los talleres del Programa de Socialización económica y proyectos de gestión de servicios turismo vivencial, centrado en rescate de los productos culturales para su comercialización, de sus expresiones culturales en paquetes turísticos

\section{Técnicas De Análisis Estadístico}

Los estadísticos empleados fueron la t de student y KR20 de Pearson para la validez y la confiabilidad de la Escala: ESCAIETA-2011

\section{Variables}

Variable dependiente: Tendencia de actitudes hacia la interdependencia para el emprendimiento.

Variable independiente: Talleres de información de términos económicos en estrategias por interdependencias empresariales o alianzas estratégicas de la gestión empresarial e institucional:

1. Taller de Organización de la unidad económica productiva de Servicio de Turismo.

2. Taller de Toma de decisión de ser miembro de la unidad económica productiva. 
3. Opción de organización de la unidad económica productiva: El Objetivo de la Asociación.

4. Taller de producción de servicios.

5. Taller de Comercialización del Servicio: Canal de Ventas.

6. Taller de Producción de una nueva línea de servicios.

7. Taller de Negociación y Gestión.

8. Taller de Costos.

9. Taller de Revaluación y retroalimentación.

Variable de Control: Grupo de asociados que realicen actividades con tareas o funciones de turismo vivencial de colaboradores de Caraz

\section{Técnicas de análisis de datos}

DATA analizada por estadígrafos no paramétricos.

\section{RESULTADOS}

\section{Resultados Descriptivos}

Según los resultados del sondeo sobre los conocimientos, ver Tabla 2, los participantes tiene un conocimiento bastante acertado sobre las necesidades del turista. Evidentemente la realidad de la infraestructura existente en los pueblos hace que el aspecto de higiene personal sea menos apreciado que el del resto de servicios, sean alimentación, información, transporte y demás.

Por otra parte, se nota confusión entre lo que es turismo rural, vivencial y de aventura. Es necesario que lleguen a conocer la diferencia pues tiene que ver con cuestiones de logística, uso de infraestructura local, mantenimiento, adecuación territorial y demás.

El turista de aventura lleva consigo todo lo que necesita o se lo proporciona el organizador, por lo que deja poco dinero en los sitios que visita: quizá contrato de guías especializados y cosas así; tal como por ejemplo que no siendo de manera expresa en el contrato del servicio, que pueda llevarse con los actores de la zona, puesto que usualmente la preparación de los servicios lo asume el mismo operador que vende el paquete turístico.

El turismo vivencial necesita un mínimo de cambios por parte de los receptores y mucha capacidad de adaptación por parte de los turistas, pues ellos van a vivir como viven los lugareños. Claro que en cuestiones de higiene personal a veces es necesario hacer algunos ajustes servicios higiénicos con cierta privacidad y limpieza en general en muebles de uso: camas, mesas, recipientes, vajilla, rechazando el plástico, privilegiando los materiales naturales (arcilla, barro, piedra, carrizo, eucalipto, pino, mate, fierro forjado) etc. 
El turismo rural sí supone una mayor inversión pues el turista espera poder disfrutar del campo pero viviendo de manera similar, salvando distancias, a cómo vive en la ciudad, esto es, tener una habitación privada, acceso a un cuarto de aseo con agua de ser posible corriente y caliente, actividades recreativas originales de la localidad: paseos a caballo, visita a granjas o viñedos, paseos a lugares paisajísticamente valiosos, baños termales o en lagunas o ríos, excursiones, etc. Además espera cocina local típica, etc. Todo ello puede ser incluido en el precio, aunque generalmente se le ofrece por separado, al turista y solo lo paga si lo usa.

Este turismo, el rural, esta también orientado con frecuencia a los jóvenes o niños, a través de organizaciones que alquilan casas o usan albergues municipales, también granjas que se dedican a ofrecer al turista vivencias del campo pero con comodidades que difícilmente tienen los campesinos.

Tabla 3: Resultados totales de la encuesta de conocimientos sobre turismo alternativo

\begin{tabular}{|c|c|c|c|}
\hline $\mathrm{N}^{\circ}$ & & Chi2 & Sign. \\
\hline 1 & $\begin{array}{l}\text { De poder viajar para conocer o disfrutar como turista ¿le gusta- } \\
\text { ría ir a un sitio igual a su pueblo o a uno diferente? }\end{array}$ & & Sí \\
\hline A & Igual a mi pueblo & 0 & \\
\hline $\mathrm{B}$ & Parecido a mi pueblo pero con algo diferente & 0.2 & \\
\hline $\mathrm{C}$ & Totalmente distinto a lo que yo conozco & $0.8^{*}$ & \\
\hline 2 & $\begin{array}{l}\text { ¿En sus viajes le gusta encontrar una cama limpia o no le impor- } \\
\text { ta que esté sucia? }\end{array}$ & & Sí \\
\hline A & Me gusta encontrar una cama y también una habitación limpia & $0.9 *$ & \\
\hline $\mathrm{B}$ & $\begin{array}{l}\text { Si la cama está limpia no me importa que la habitación esté algo } \\
\text { sucia. }\end{array}$ & 0 & \\
\hline $\mathrm{C}$ & Me da igual cómo esté la cama o la habitación & 0.1 & \\
\hline 3 & Cuando viajo me gusta encontrar: & & Sí \\
\hline A & Comida fresca y bien preparada & $0.9 *$ & \\
\hline $\mathrm{B}$ & No me importa la comida & 0 & \\
\hline $\mathrm{C}$ & Prefiero llevar mi comida cuando viajo por varios días & 0.1 & \\
\hline 4 & Cuando viajo me gusta encontrar: & & Sí \\
\hline A & Un lugar privado donde asearme dentro de la casa donde me alojo & $0.7 *$ & \\
\hline $\mathrm{B}$ & Me es suficiente un caño de agua y un servicio afuera de la casa & 0.2 & \\
\hline $\mathrm{C}$ & Con una batea de agua y el campo tengo bastante & 0.1 & \\
\hline 5 & Si voy a pasear por un sitio que no conozco espero encontrar: & & Sí \\
\hline A & Señales que me orienten para no perderme & $0.8^{*}$ & \\
\hline $\mathrm{B}$ & Personas que lo conozcan y me acompañen & 0.2 & \\
\hline $\mathrm{C}$ & Me gusta aventurarme y arriesgarme a perderme & 0 & \\
\hline 6 & Si voy a pasear por un sitio que no conozco: & & Sí \\
\hline A & $\begin{array}{l}\text { Me gusta conocer los lugares, las costumbres y tradiciones de ese } \\
\text { sitio. }\end{array}$ & $0.9 *$ & \\
\hline $\mathrm{B}$ & Me gusta conocer cómo viven las personas de ese sitio & 0.1 & \\
\hline
\end{tabular}




\begin{tabular}{|c|c|c|c|}
\hline $\mathrm{C}$ & Me da lo mismo & 0 & \\
\hline 7 & Si viajando me enfermo o me accidento: & & \\
\hline A & Quiero tener asistencia médica inmediata & $0.8^{*}$ & \\
\hline $\mathrm{B}$ & Puedo esperar a que me atiendan cuando puedan & 0 & \\
\hline $\mathrm{C}$ & Siempre llevo mis propias medicinas & 0.2 & \\
\hline 8 & Para conocer y pasear por los lugares a los que viajo: & & No \\
\hline A & Me gusta encontrar carros que me lleven & 0.2 & \\
\hline $\mathrm{B}$ & Me gusta que haya caballos para ir por caminos diferentes & 0.1 & \\
\hline $\mathrm{C}$ & Me gusta ir en bicicleta a mi manera & 0 & \\
\hline $\mathrm{D}$ & Me gusta caminar & 0.1 & \\
\hline $\mathrm{E}$ & Todo lo anterior & 0.6 & \\
\hline 9 & Cuando visito un sitio: & & No \\
\hline A & $\begin{array}{l}\text { Me gusta que haya explicaciones sobre qué es y lo que significa para } \\
\text { los pobladores de la zona }\end{array}$ & 0.6 & \\
\hline $\mathrm{B}$ & No me interesa conocer sobre el sitio & 0 & \\
\hline $\mathrm{C}$ & $\begin{array}{l}\text { Me agradan las historias o cuentos propios o misterios que tiene el } \\
\text { pueblo }\end{array}$ & 0.4 & \\
\hline 10 & $\begin{array}{l}\text { El turismo rural y el turismo vivencial, conocido también como } \\
\text { turismo alternativo tiene; }\end{array}$ & & No \\
\hline A & Ordenanza municipal & 0.3 & \\
\hline $\mathrm{B}$ & Tiene ley reglamentada & 0.4 & \\
\hline $\mathrm{C}$ & No tiene & 0.3 & \\
\hline 11 & $\begin{array}{l}\text { La diferencia entre turismo rural, turismo vivencial, turismo } \\
\text { tradicional y turismo de aventura es: }\end{array}$ & & Sí \\
\hline A & $\begin{array}{l}\text { En el Turismo Rural y el Vivencial el visitante se aloja en una casa } \\
\text { particular, en el Tradicional se aloja en un hotel y el de aventura en } \\
\text { un campamento }\end{array}$ & $0.8^{*}$ & \\
\hline $\mathrm{B}$ & $\begin{array}{l}\text { En el Turismo Rural los visitantes se pasean por el campo, en el de } \\
\text { aventura se pasean por lugares peligrosos y en el tradicional por la } \\
\text { ciudad y monumentos }\end{array}$ & 0.1 & \\
\hline $\mathrm{C}$ & $\begin{array}{l}\text { El Turismo Rural y el Vivencial dan plata al campo y el Tradicional } \\
\text { y de Aventura dan plata a la ciudad }\end{array}$ & 0.1 & \\
\hline 12 & Turismo rural es: & & No \\
\hline A & $\begin{array}{l}\text { Ir a una ciudad, alojarse en un hotel, comer en restaurantes y pasear } \\
\text { por la ciudad y alrededores }\end{array}$ & 0.1 & \\
\hline $\mathrm{B}$ & $\begin{array}{l}\text { Ir a un pueblo, alojarse en una casa particular y vivir unos días des- } \\
\text { cansando y disfrutando de los alrededores y de la vida del pueblo }\end{array}$ & 0.4 & \\
\hline $\mathrm{C}$ & $\begin{array}{l}\text { Compartir con los habitantes de un sitio las actividades que realizan } \\
\text { día a día }\end{array}$ & 0.5 & \\
\hline 13 & Turismo vivencial es: & & Sí \\
\hline
\end{tabular}




\begin{tabular}{|c|c|c|c|}
\hline A & $\begin{array}{l}\text { Ir a una ciudad, alojarse en un hotel, comer en restaurantes y pasear } \\
\text { por la ciudad y alrededores }\end{array}$ & 0 & \\
\hline $\mathrm{B}$ & $\begin{array}{l}\text { Ir a un pueblo, alojarse en una casa particular y vivir unos días des- } \\
\text { cansando y disfrutando de los alrededores y de la vida del pueblo }\end{array}$ & 0.2 & \\
\hline $\mathrm{C}$ & $\begin{array}{l}\text { Compartir con los habitantes de un sitio las actividades que realizan } \\
\text { día a día }\end{array}$ & $0.8^{*}$ & \\
\hline \multirow[t]{3}{*}{ 14A } & $\begin{array}{l}\text { Turismo rural es compartir con los habitantes de un sitio las } \\
\text { actividades que realizan día a día. }\end{array}$ & & Sí \\
\hline & Correcto & 0.3 & \\
\hline & Incorrecto & $0.7 *$ & \\
\hline \multirow[t]{3}{*}{ 14B } & $\begin{array}{l}\text { Turismo de aventura: ir a un pueblo, alojarse en una casa parti- } \\
\text { cular y vivir unos días descansando y disfrutando de los alrede- } \\
\text { dores y de la vida del pueblo }\end{array}$ & & Sí \\
\hline & Correcto & $(0.9 *)$ & \\
\hline & Incorrecto & 0.1 & \\
\hline \multirow[t]{3}{*}{$14 \mathrm{C}$} & $\begin{array}{l}\text { Turismo tradicional: pasear por lugares peligrosos a pie o en } \\
\text { algún tipo de vehículo }\end{array}$ & & Sí \\
\hline & Correcto & $(1 *)$ & \\
\hline & Incorrecto & 0 & \\
\hline \multirow[t]{3}{*}{ 14D } & $\begin{array}{l}\text { Turismo vivencial: ir a una ciudad, alojarse en un hotel, comer } \\
\text { en restaurantes y pasear por la ciudad y alrededores }\end{array}$ & & Sí \\
\hline & Correcto & $(0.9 *)$ & \\
\hline & Incorrecto & 0.1 & \\
\hline \multirow[t]{3}{*}{$15 \mathrm{~A}$} & El dinero que genera el turismo rural es para la ciudad & & Sí \\
\hline & Verdadero & $\left(1^{*}\right)$ & \\
\hline & Falso & 0 & \\
\hline \multirow[t]{3}{*}{ 15B } & $\begin{array}{l}\text { En el turismo vivencial, el turista se limita a pasear por las calles } \\
\text { del pueblo }\end{array}$ & & Sí \\
\hline & Verdadero & $(1 *)$ & \\
\hline & Falso & 0 & \\
\hline \multirow[t]{3}{*}{$15 \mathrm{C}$} & El turista de aventura se aloja en las casas del pueblo & & Sí \\
\hline & Verdadero & $(1 *)$ & \\
\hline & Falso & 0 & \\
\hline \multirow[t]{3}{*}{ 15D } & Con el turismo tradicional gana dinero la ciudad & & Sí \\
\hline & Verdadero & $1^{*}$ & \\
\hline & Falso & 0 & \\
\hline
\end{tabular}

Se presenta entre paréntesis los criterios en que los encuestados se eaquivocan

$*=$ Sign al 0,05 


\section{Resultados Cualitativos}

Aplicación de la VI, Taller: Opción de organización de la unidad económica productiva: El Objetivo de la Asociación. A propósito de formular objetivo de una Unidad productiva, se reinició con un diagnóstico de expectativas, que se presentan a continuación de uno de los grupos cuasi experimentales, teniéndose como técnica la dinámica de grupo en su modalidad de focus group.

Consolidado 1: ¿qué esperanzas tengo en torno a la Formación de la Unidad Productiva?: tiene por resultados que requieren Capacitación, financiamiento, participación.

Tabla 4: Planteamientos por los Asociados de APEMYPE Caraz. 12 de junio 2011

Preguntas Equipo Huandoy Grupo El Amanecer Grupo Caraz $\begin{gathered}\text { Empresa Las } \\ \text { Delicias De } \\ \text { Caraz }\end{gathered}$

1. ¿Que esperanza tengo en la unidad productiva?
Crecer, prosperar, mejorar la atención al turista, la capacidad instalada y estar acorde a la competencia y lograr el éxito, y dar una buena atención y servicio al usuario.

Mejorar el servicio al usuario

Modernizar la infraestructura para dar al usuario una buena atención.

Concretar la construcción de un hostal.

Adecuar los servicios de acuerdo a las necesidades del usuario.

Convertir mi negocio en un complejo turístico rustico elegante, con todo los avances modernos.

Que en base a los principios o valores de responsabilidad y honestidad tengamos una unidad productiva desarrolla-

El desarrollo socioeconómico de la ciudad de Caraz.

Cambio de políticas en turismo.

Capacitación y desarrollo productivo sostenible.

Fortalecimiento de las organizaciones públicas y privadas.

Crear microempresa en bien del turismo.
Habría más empleo.

De crecer y generar más empleo

De generar más economía en la ciudad de Caraz

Ampliar la cebichería. da y moderna,

acorde a la

tecnología

y tener una productividad óptima.

Esperanza de contar con el apoyo técnico y el financiamiento o apoyo económico para hacer realidad el proyecto.

$$
\begin{aligned}
& \text { Mejorar la cali- } \\
& \text { dad de nuestros } \\
& \text { productos para } \\
& \text { una buena aten- } \\
& \text { ción al mercado } \\
& \text { nacional y } \\
& \text { posteriormente } \\
& \text { internacional. }
\end{aligned}
$$


2. ¿Que esperanza tengo en los asociados?
Que los socios tomen mayor conciencia en el compromiso con su tierra.

Una ayuda mutua entre todos los asociados.

Que los socios deben trabajar con competencia leal.

Que haya lealtad en el apoyo económico y ver el progreso en Caraz.

Que todos los socios siempre trabajemos unidos y sacar adelante nuestros negocios.
La esperanza del apoyo para poder progresar en el negocio que tenemos.

Trabajo.

Reciprocidad.

Meter el hombro mancomunadamente.

Lealtad.

Cultivar los valores y principios.

La esperanza de progresar económicamente en Caraz.

Que entre todos nos apoyemos, para poder crecer como empresarios contando con el apoyo y capacitación constante de la APEMIPE
Tener confianza con los asociados para poder actuar con responsabilidad, respeto y honestidad.

Encontrar cooperación mutua como asociados

Que se actúe con ética y no sé de una competencia desleal.
3. ¿Que esperanza tengo en torno a la formacion de la unidad productiva?
Mejorar Caraz y la condición de vida.

Que nuestra unidad productiva atienda bien al turista y lograr que regresen.

Buscar orientación para lograr el desarrollo de nuestra empresa.

Mi esperanza dentro del APEMIPE es lograr que nos capaciten $\mathrm{y}$ financien nuestros negocios y salir adelante.
Que nuestro

Formar un buen sector se le negocio para dé la imporpoder tener una tancia y se Voluntad de los miem- buena producbros.

\section{Participación}

Firmeza y consecuencia.

Identidad.

Realista no soñar. ción.

Que estén bien capacitados e instruidos para prestar un buen servicio

Que el ambiente laboral sea óptimo para un buen desempeño laboral. haga realidad nuestros proyectos a presentarse.

Incrementar los asociados de nuestra localidad con ideas de futuro y poderlos constituir legalmente. 
4. ¿Que productos puedo ofrecer para la venta?
Platos típicos de la

Zona: Cuy (Acacashqui, picante, pachamanca); Pachamanca 5 sabores; Truchas

Ofrecer platos típicos que ya están desapareciendo como caldo, estofado, acacasqui, llunca de gallina, cuy, estofado de gallina.

Ofrecer ambientes con material de la zona y platos ricos.

Hostal, ofrecer buenas camas con habitaciones adecuadas para el usuario.

Discoteca. Ofrecer buenos tragos, buena música, y buen servicio.

(Chicharrón, sudado, ceviche, jaleas);

Pescados y mariscos

(Ceviche, jugoso,

chicharrón, jalea, arroz con marisco, la Caraz);

La quiwicha la mashwa.

Recursos naturales, históricos, culturales, vía Internet y otros medios (Agencias, radio, tv, trípticos, volantes).

La hospitalidad, calicreación del ceviche en dez de nuestra gente.

Servicios básicos.

Platos típicos de la zona como de la sierra, selva y costa

Bebidas típicas del Perú

Comidas típicas de la región, variedad de cafés, sándwich, pitzas; variedad de platos marinos y según el gusto del cliente.

Manjar blan-

co, chancaquitas, cuarteados alfajores.

Miel de abeja, polen, jalea real, propolios, polimiel, cuatro en uno.

Crianza de abejas, colmenas.

Crear una página web ofreciendo nuestros productos para una mejor venta.

Nuestras costumbres e identidad.

Consolidados 2: Tiene por resultados que requieren el FORTALECIMIENTO DE LA ASOCIACIÓN SHOCSHA VIVENCIAL.

Tabla 5: Planteamientos por los asociados de la asociación shocsha vivencial.

Respuestas

Tratar directamente con los turistas sin intermediarios.

Debemos ser capacitados y reorganizados como aso-

¿Que esperanza tengo en la unidad

ciación.

productiva?

Dar fortaleza a nuestra organización

Reorganización de una esperanza para organizar fuentes de trabajo

¿Que esperanza tengo en los asociados

Que tenga fortaleza como asociación por los asociados. de shocsha?

Que los asociados sean más responsables 
Tenemos un clima adecuado.

Tenemos flora y fauna.

¿Que esperanza tengo en torno a la formacion de la unidad productiva de

Tenemos animales exóticos shocsha?

Tenemos criaderos y pequeñas granjas.

Áreas de cultivo.

Todo tipo de cereales y legumbres.

Venta de miel.

¿Que productos puedo ofrecer para la venta?

Venta de hortalizas.

Venta de mermeladas.

Frutas de la zona.

\section{Resultados cuantitativos}

Se encuentra que en la población de colaboradores presentan los siguientes resultados, los que pueden observar en la Tabla 4:

Las actitudes hacia la interdependencia empresarial para el turismo en la ciudad de Caraz se encuentran en el nivel de Positivo.

La intensidad de la actitud hacia la en interdependencia empresarial antes de la aplicación del programa es igual a 2.91 y después del programa es 3.14, ambas en el grado 3 de una escala de 4 grados.

No se encuentra diferencia significativa entre antes y después de la aplicación de la condición experimental.

Tabla 6: Comparación de medias antes y después de las actitudes hacia la interdependencia empresarial para el emprendimiento

\begin{tabular}{rcc}
\hline & ANTES & DESPUES \\
\hline Media & 2.56 & 3.32 \\
Varianza & 0.43 & 1.43 \\
$\mathrm{n}$ & 10 & 10 \\
\hline Diferencia & & 0.75 \\
$\mathrm{t}$ & & 0.38 \\
& & 2.00 \\
\hline
\end{tabular}




\section{DISCUSIÓN}

La población participante es conocedora de las características que hacen de su ciudad un destino turístico atractivo, apto para diferentes tipos de turismo, pero más interesante para el de aventura, vivencial y rural, con paisajes variados y todos muy reconocidos por su belleza. También identifican correctamente la mayoría de exigencias que plantean los turistas con respecto a su confort y las metas de su viaje. Estos puntos son adecuados para la meta perseguida por la intervención, pero hay otros resultados que refutan la hipótesis principal y que muestran las debilidades de la población en su aproximación al turismo alternativo en sus modalidades de rural y vivencial.

En el sentido que el Programa de Socialización Económica en Interdependencias Empresariales e Institucionales para orientar el Emprendimiento del Turismo RuralVivencial en Caraz, Ancash, no tiene efectividad, es atribuible al conocimiento o percepción de los logros que tienen las agencias que se dedican al turismo de aventura, así como a que sus actividades para el sector turismo no les reditúa, además de que los productos de sus expresiones culturales comercializables son escasos. Los que tienen más éxito en este negocio por lo general son foráneos, mientras que los del lugar logran escaso valor de los consumidores.

La falta de claridad se refiere más a lo conceptual que a lo emocional. En el primer aspecto esta población tiende a confundir el turismo rural con el vivencial tanto en sus características, como según el tipo de turismo que les posibilita acceder a lograr mejores y mayores utilidades y para quien se constituye una fuente de ingreso, es decir sobre todo aquel tipo del sector turismo es el mayor beneficio, pues en una de sus respuestas señalan que el dinero generado por el turismo rural se va a la ciudad, lo que demuestra que en la práctica podría ser así, ya que ven circular y venir turistas que recorren su territorio, con sus respectivos enseres o provisiones.

Al respecto se puede observar lo siguiente, que es coincidente con las en investigaciones del mismo tema en otros lugares, donde se halla resultados similares, en los que al menos una parte de la población se manifiesta poco interesada por el turismo, probablemente en la medida en que el turismo es incapaz de alcanzar beneficios directos a los pobladores, como en el caso de la ruta del vino en Inglaterra.

Según Allen y otros (1993), las actitudes dependen de la relación entre la condición económica y el desarrollo del turismo, pero debe precisarse que el turismo en Caraz tiene un desarrollo incipiente y la población es de bajos recursos, observándose una actitud favorable pero no muy alta hacia el emprendedorismo turístico mediante la formación de articulaciones económicas.

Otro aspecto a considerar es que la tendencia a la resistencia al cambio es mayor que la predisposición a entenderlo, además de que el turismo en Caraz es más bien estacional y poco canalizable por los lugareños, pues los visitantes en realidad hacen pocos gastos en la ciudad misma, llegan ya abastecidos, en razón del tipo de actividad que van a realizar, protección a su salud, por los efectos que pudiera 
tener un alimento específico, incluida el agua y por qué las agencias de turismo tratan de copar todos los aspectos. Aquí la historia del foráneo que llega y se vuelve un triunfador es una especie de recorte de oportunidades que sufre el lugareño.

\section{CONCLUSIONES}

1. No existe diferencia significativa entre antes y después de la aplicación del Programa de Socialización Económica en Interdependencias Empresariales e Institucionales para Orientar el Emprendimiento del Turismo Rural-Vivencial en Caraz, Ancash.

2. Las actitudes hacia la Interdependencia empresarial para el emprendimiento son positivas (3.32 sobre una escala de 4 grados).

3. Se observa una tendencia al incremento de la actitud hacia la interdependencia empresarial para el emprendimiento, sin llegar a marcar una diferencia significativa ni a pasar a muy positiva. Pasó de 2.56 a 3.32 sobre una escala de 4 grados.

4. Se encuentra confusión sobre las modalidades del turismo.

5. Claridad en su opinión y expectativas sobre la importancia del turismo Alternativo: Rural-vivencial.

\section{REFERENCIAS BIBLIOGRÁFICAS}

Allen, L. R., Hafer, H. R., Long, P. T., \& Perdue, R. R. (1993). Rural residents' attitudes toward recreation and tourism development. Journal of Travel Research, 31(4), 27-27. Retrieved from http://search.proquest.com/docview/217443547?accountid $=12268$

Ardila, R. (1993) Psicología Social de la Pobreza. En Whittakker, J. O. La psicología social en el mundo de hoy. (399-418) Edit. Trillas.

Azerbaijani MP voices attitude towards law on tourism (2011). Azerbaijan, Baku: Trend News Agency. Retrieved from http://search.proquest.com/docview/885455938?acc ountid $=12268$

Blanchard, K., Carlos J., Randolph. (2002). Las 3 claves para el empowerment. Cómo dar poder para liberar la capacidad de las personas y lograr que den lo mejor de sí. Barcelona: Ediciones Granica.

Bregman, P (2009). Cómo contrarrestar la resistencia al cambio. Harvard Business Review America Latina, Obtenido el 19 de Noviembre de 2010, en http://www.hbral.com/ blog $/$ blog. asp? modulo $=2 \&$ idBlog $=35$

Denegri, Marianela; Gempp, Rene; Del Valle, Carlos; Etchebarne, Soledad; González, Yessica. (2006). Aporte de la psicología educacional a las propuestas de educación económica: los temas claves. Revista de Psicología. Universidad de Chile. Chile. Vol. XV, $\mathrm{N}^{\mathrm{o}} 2$ 
Denegri, Coria Marianela, Del Valle R. Carlos; Gempp Fuentealba, René y Lara Arbola; Miguel A. (2006) Educación Económica en la escuela: hacia una propuesta de intervención. Chile Estudios Pedagógicos XXXII, No 2: 103-120, 2006.

Denegri, C. M. y Equipo Proyecto de Investigación , 2007. "Yo y la Economía”: Diseño, Aplicación y Evaluación de impacto de un Programa de Educación Económica en Escolares de $6^{\circ}$ Año Básico de Temuco. UFRO, Chile

Denegri, M., Fernández, F., Mireya I., Ripio, M., (1999) Consumir para vivir y no vivir para consumir. Temuco, Chile. Ediciones Universidad de La Frontera.

Gursoy, D., Chi, C. G., \& Dyer, P. (2010). Locals' attitudes toward mass and alternative tourism: The case of Sunshine Coast, Australia. Journal of Travel Research, 49 (3), 381. Retrieved from http://search.proquest.com/docview/749481577?account id $=12268$

Ho, P. (2011). An empirical study of residents' attitudes to tourism impact management strategies. Journal of American Academy of Business, Cambridge, 17(1), 232232-238. Retrieved from http://search.proquest.com/docview/870507791?account $\mathrm{id}=12268$

Howley, M., \& Westering, J. v. (2008). Developing wine tourism: A case study of the attitude of english wine producers to wine tourism. Journal of Vacation Marketing, 14(1), 87-87-95. Retrieved from http://search.proquest.com/docview/195819943?a ccountid $=12268$

Paredes, M. y Colb. (2010 A) Programa de Alfabetización Económica sobre conductas de emprendimiento. Informe Técnico CSI 2010- Proy. 1018 01011-2010, UNMSM/ CSI/IPPSI.

Paredes M, y Cob. (2009 B) Informe Técnico Proyecto No. Código: 091801061: Socialización económica para el empoderamiento del caserío como unidad económica de la articulación organizacional de la Comunidad Campesina Cruz de Mayo, Caraz. CSI/UNMSM.

Paredes, T. Mildred, 2009. El caserío es la unidad productiva en la articulación organizacional de zonas rurales altoandinas, Callejón de Huaylas, Ancash. Revista del Instituto de Investigaciones Económicas, Pensamiento Crítico, N. ${ }^{\circ}$ 11:73-96, Junio, Lima.

Paredes T., M; Hernández, H; Vicuña L; Rivera, J C.; Arias A. V; (2009) Percepción del conocimiento de las expresiones culturales y su revalorización integral en dos comunidades altoandinas en Ancash, Lima, Perú. Revista de Investigación en Psicología, UNMSM / Facultad de Psicología.

Paredes T., M.; Hernández, H. Vicuña, L. De Miguel, J Ma., (2007). Percepción del conocimiento de la demanda de productos y percepción hacia la interdependencia económica en el Caserío Queral, Caraz, Ancash, Revista de Investigación en Psicología, Vol. 10 N. ${ }^{\circ}$ 1:109-124, UNMSM / Facultad de Psicología

Paredes, M. y De Miguel, J. Ma . (2004). Construcción social de la comunidad para el Desarrollo del Callejón de Huaylas, Caraz. Revista de Investigación en Psicología, UNMSM / Facultad de Psicología. Vol. 7 N. ${ }^{\circ} 1,81-102$. 
Paredes T., M.; De Miguel, J. Ma.; Vicuña, L. y Hernández, H. (2005). El papel de la construcción social de la organización con respecto a la percepción de las condiciones de trabajo y el manejo del medio ambiente en 4 caseríos de una comunidad campesina andina - Caraz, Ancash Vol.8 N. ${ }^{\circ} 16$ Revista del Instituto de Investigación, Facultad de Ingeniería Geológica, Minería, Metalúrgica y Geografía, UNMSM. ISSN.

Paredes, M. Vicuña, L. y Hernández, H. 2002, Formación de actitudes para el éxito en la carrera de jockey. Revista de Investigación en Psicología, Vol. 5, N. ${ }^{\circ}$ 2, 71-86. U.N.M.S.M. / Facultad de Psicología

Plan Estratégico de Desarrollo Concertado de la Provincia de Huaylas 2007-2015 
